\title{
Operation modes of the FALCON ion source as a part of the AMS cluster tool
}

Oleksii Girka, Alexander Bizyukov, Ivan Bizyukov, Michael Gutkin, Sergei Mishin

\begin{abstract}
$\overline{\text { Abstract. The paper investigates the options to increase the production yield of temperature compensated surface }}$ acoustic wave (SAW) devices with a defined range of operational frequencies. The paper focuses on the preparation of large wafers with $\mathrm{SiO}_{2}$ and $\mathrm{AlN} / \mathrm{Si}_{3} \mathrm{~N}_{4}$ depositions. Stability of the intermediate $\mathrm{SiO}_{2}$ layer is achieved by combining high power density UV radiation with annealing in high humidity environment. A uniform thickness of the capping AlN layer is achieved by local high-rate etching with a focused ion beam emitted by the FALCON ion source. Operation parameters and limitations of the etching process are discussed.
\end{abstract}

Key words: FALCON • ion source $\bullet$ surface wave $\bullet$ cluster tool

O. Girka ${ }^{\bowtie}$, A. Bizyukov, I. Bizyukov

V. N. Karazin Kharkiv National University,

4 Svobody Sq., 61022, Kharkiv, Ukraine,

E-mail: oleksiigirka@karazin.ua

\section{Gutkin}

Micron Surface Technologies,

5033 Dantes View Drive, Calabasas, CA 91301, United States

\section{S. Mishin}

Advanced Modular Systems, Inc,

354 South Fairview Ave., B1, Goleta, CA 93117,

United States

Received: 19 May 2014

Accepted: 5 May 2015

\section{Introduction}

Improvement of frequency uniformity and stability for surface acoustic wave (SAW) devices [1] is an important technological task, a solution of which requires advanced methods. Currently, SAW devices are widely used in mobile communication devices as filters based on converting electrical signals to acoustic waves and vice versa by exploiting the piezoelectric effect. These devices are fabricated by photolithography. However, the initial stage of manufacturing SAW devices requires large $\mathrm{Si}$ wafers to be covered with a $\mathrm{SiO}_{2}$ intermediate layer and a capping layer consisting of silicon nitride $\left(\mathrm{Si}_{3} \mathrm{~N}_{4}\right)$ or aluminum nitride (AlN), see Fig. 1. The thickness of the capping layer and its bulk properties define the frequency of the SAW device. It is highly desirable to keep the scattering of operational frequencies of SAW devices, obtained during the manufacturing process, as low as possible to maximize the frequency uniformity. Also, the intermediate $\mathrm{SiO}_{2}$ layer should stabilize the initial properties of the capping layer.

$\mathrm{SiO}_{2}$ has been used to obtain a low temperature coefficient (TempCo) in SAW devices for more than three decades $[2,3]$. One of the big issues is that SAW devices have to be processed at temperatures below $300^{\circ} \mathrm{C}$. When $\mathrm{SiO}_{2}$ is exposed to air at low temperature during the manufacturing process, the frequency of the SAW devices may change [4]. Diffusion of the absorbed water molecules into the $\mathrm{SiO}_{2}$ layer causes a reaction of $\mathrm{H}_{2} \mathrm{O}$ with strained $\mathrm{Si}-\mathrm{O}$ bonds and formation of silanol $(\mathrm{Si}-\mathrm{OH})$. This has a big impact on the local intrinsic $\mathrm{SiO}_{2}$ layer stress, leads to the reduction of compressive residual stress 


\section{Capping layer}

$\mathrm{SiO}_{2}$

\section{Si wafer}

Fig. 1. A schematic cross section of the wafer with depositions before manufacturing SAW devices with lithography.

and a progressive increase of the tensile residual stress.

Another issue is the choice of the capping layer material and its surface processing. Using silicon nitride $\left(\mathrm{Si}_{3} \mathrm{~N}_{4}\right)$ as the capping layer on top of $\mathrm{SiO}_{2}$ showed some improvement in the frequency uniformity after trimming. Up to now, the best results were obtained using an aluminum nitride (AIN) capping layer on top of $\mathrm{SiO}_{2}$. While uniform deposition of the $\mathrm{SiO}_{2}$ layer (small variation of the layer thickness over the wafer) is a well-established technological process, uniform deposition of the capping layer is not available at the moment and its thickness varies strongly over the wafer. Therefore, one of the key operations in the production of SAW devices is the trimming process $[5,6]$, which utilizes local high-rate etching combined with a focused ion beam. It decreases the local thickness of the layer achieving uniform thickness of the capping layer over the wafer and, consequently, improves the frequency uniformity.

This paper discusses the options available for improving the frequency uniformity of the SAW devices and their particular technological implementations.

\section{Experimental setup}

We used an Advanced Modular Systems' (AMSystems) three chamber cluster tool [1] for deposition of all layers as well as for trimming. The cluster tool allows transport of the processed wafer from one chamber to another without exposure of its surface to the air, i.e. all chambers and connecting volumes are maintained at pressures below $10^{-6}$ Torr.

In one of the chambers, $\mathrm{SiO}_{2}$ is deposited using the RF diode mode from 12 inch $\mathrm{SiO}_{2}$ target at $1 \mathrm{~kW}$ of RF power and a frequency of $13.56 \mathrm{MHz}$. Only Ar process gas was added during $\mathrm{SiO}_{2}$ deposition. Another chamber was used for deposition of the capping layer. $\mathrm{A} \mathrm{Si}_{3} \mathrm{~N}_{4}$ layer was deposited by a dual alternative current (AC) magnetron from a highly doped and highly conductive Si target. $40 \mathrm{kHz}$ AC power at $1 \mathrm{~kW}$ was applied between two Si targets. $\mathrm{Ar}$ and $\mathrm{N}_{2}$ were added as process gases during deposition. Deposition was done in poisoned nitride mode to get a stable and stoichiometric $\mathrm{Si}_{3} \mathrm{~N}_{4}$ layer. The standard process for AlN deposition includes the application of a dual AC magnetron operated in a reactive deposition mode.

The third chamber had been used for the trimming process, which was performed on the deposited layers. The process is established by local sputtering of the capping layer using an adjustable focused direct current (DC) ion source and the scanning wafer mechanism. The trimming process was applied to obtain a higher production yield of devices from a wafer. As it has been mentioned above, two materials, namely AlN and $\mathrm{Si}_{3} \mathrm{~N}_{4}$, were investigated as cap layers in order to prevent physical adsorption of water molecules on the silicon dioxide layer. Both layers have good passivation properties. They are stable and have a high grain density. Layers of $\mathrm{Si}_{3} \mathrm{~N}_{4}$ or AlN with $500 \AA$ thickness were deposited on top of the $\mathrm{SiO}_{2}$ layers, providing continuous vacuum conditions during the technological processes. A thickness of the capping layer of $500 \AA$ was chosen so as to assure that a minimum layer thickness of $200 \AA$ remains after the trimming process.

\section{Results and discussion}

For the $\mathrm{SiO}_{2}$ surface modification we used two methods:

1. High power density UV radiation;

2. Annealing of $\mathrm{SiO}_{2}$ film in high humidity environment.

High power density UV irradiation was done by scanning the wafer with an excimer laser radiation. An XeCl excimer laser operating at a wavelength of $308 \mathrm{~nm}$ and delivering a power density greater than $10 \mathrm{MW} / \mathrm{cm}^{2}$ per pulse was used for $\mathrm{SiO}_{2}$ surface modification. Surface densification under these processing conditions prevented diffusion of the moisture into $\mathrm{SiO}_{2}$ grain bonds. The annealing of $\mathrm{SiO}_{2}$ film in high humidity was done at temperatures between 250 and $300^{\circ} \mathrm{C}$ in water steam, with post-annealing and later drying. This process allows saturating all available Si-O bonds and eliminating further changes under exposure to the ambient.

The most successful method is to cap the $\mathrm{SiO}_{2}$ layer with AlN and a subsequent double-step trimming process. The layer is etched using the FALCON ion source [7], which is essentially an anode layer thruster with a focused ion beam [8]. It is based on the design of closed drift thrusters (also known as Hall thrusters), which are typically used for space propulsion due to their compactness, simplicity and very high efficiency of converting inlet gas molecules and atoms into ions of the beam [9].

The ion beam current is primarily defined by the gas type and its flow through the discharge gap. Figure 2 shows the increase of ion current produced by the FALCON ion source with increasing pressure in the vacuum chamber, which in turn is defined by the increasing gas flow through the system. One can see that the most efficient operation of the ion source is obtained in the pressure range of $(1-4) \times 10^{-4}$ Torr. Increasing pressure further by increasing the gas flow leads to a transition of the gas discharge towards the magnetron type resulting in a subsequent deformation 


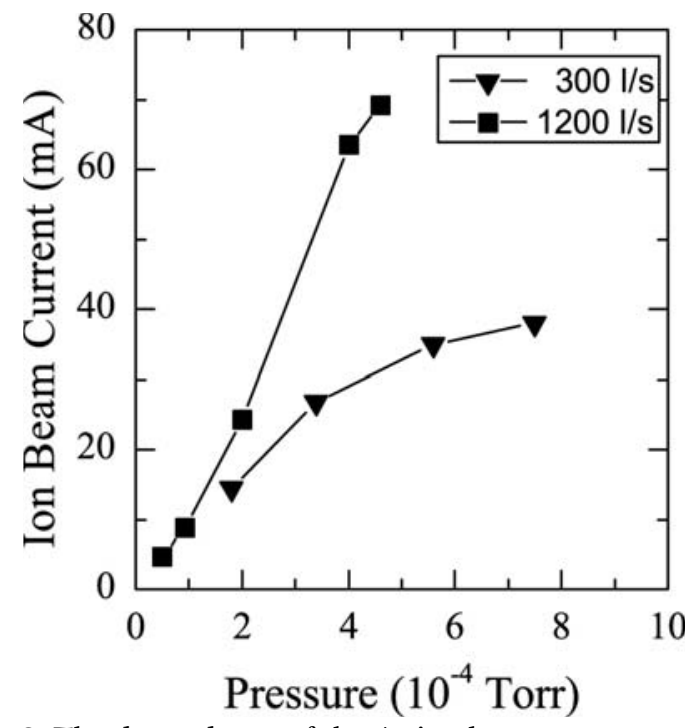

Fig. 2. The dependence of the Ar ion beam current on the vacuum chamber pressure obtained at lower and higher pumping speed. The accelerating voltage was $5 \mathrm{keV}$.

of the beam profile, enhanced erosion of cathodes, etc. Obviously, higher pumping speeds allow higher ion beam current and higher etching rate to be obtained in the optimal pressure range.

Another important parameter is the average ion energy, which defines the sputtering yield for the given pair of projectile-target combination and, consequently, the etching rate. By increasing the applied voltage and, consequently, the average ion energy, the ion beam current increases, as shown in Fig. 3. Higher pumping speeds provide higher ion beam currents at the given acceleration voltage, which is especially important for lower acceleration voltages.

Both parameters, ion beam current and ion energy, define the etching rate, but at the same time they define also the total power of the beam and the power transferred to the processed surface. Therefore, it is important to maintain higher etching rates preventing overheating of the processing wafer. While active cooling is possible, this solution is very expensive; application of higher pumping speeds and lower ion energies is preferential for the trimming process.

It has been proven that further benefit is achieved by applying a second trimming process. This allows for a further decrease in the variation of the layer thickness over the wafer and, therefore, for an increase in the production yields of the SAW devices. Figure 4 shows an example, how the frequency distribution of the manufactured SAW devices varies depending on the application of the trimming process. If the layers are deposited without a trim, a wide range of frequencies for the final SAW devices is obtained. Applying a first trim, one can achieve significant increase of the production yield for SAW devices from 120 to 250 units with an operation frequency around $1848 \mathrm{MHz}$. Moreover, applying a second trim, one can achieve a further increase of the yield from 500 to $1100-1200$ units. Depending on the initial frequency distribution, the first trim can increase the production rate by a factor of 5 , while the second trim provides another improvement factor of 4 compared to the first trim. As a result,
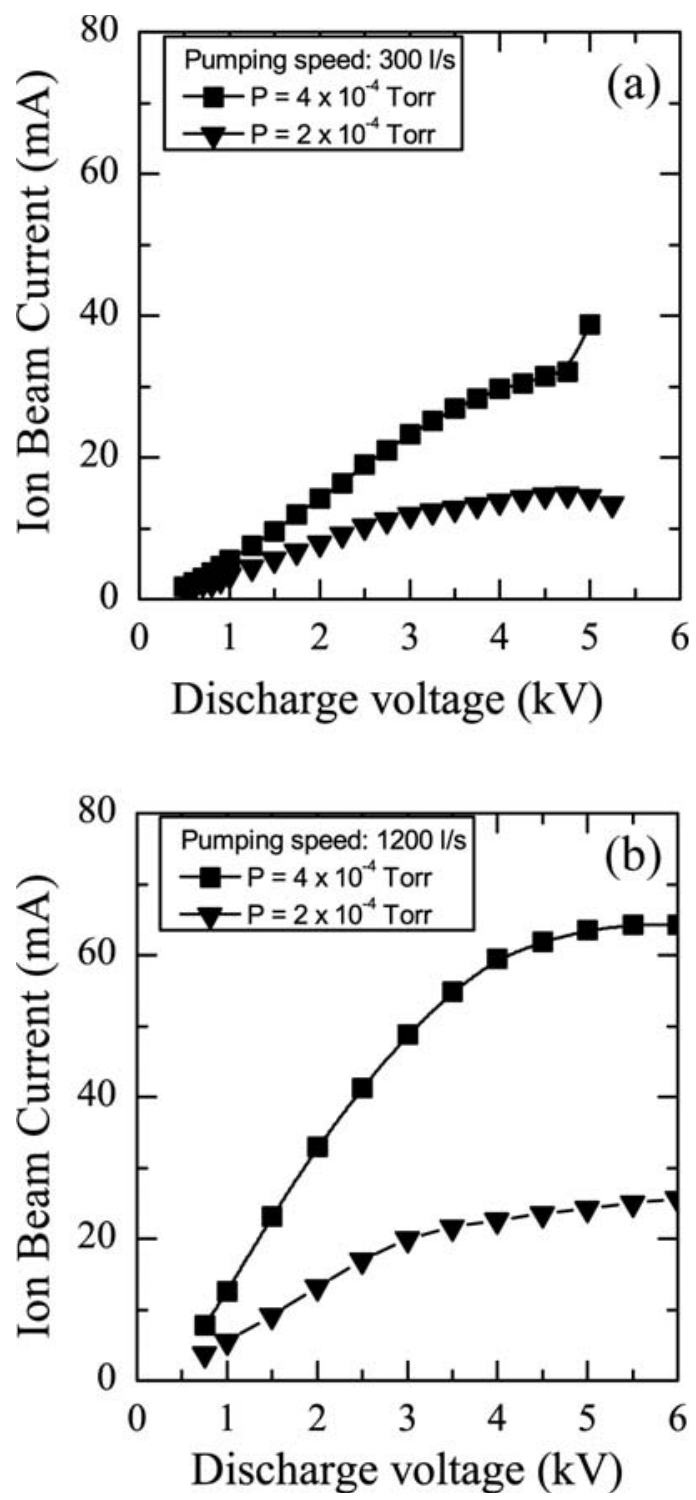

Fig. 3. The total Ar ion beam current as a function of the accelerating voltage: (a) pumping speed $300 \mathrm{l} / \mathrm{s}$; (b) pumping speed $1200 \mathrm{l} / \mathrm{s}$.

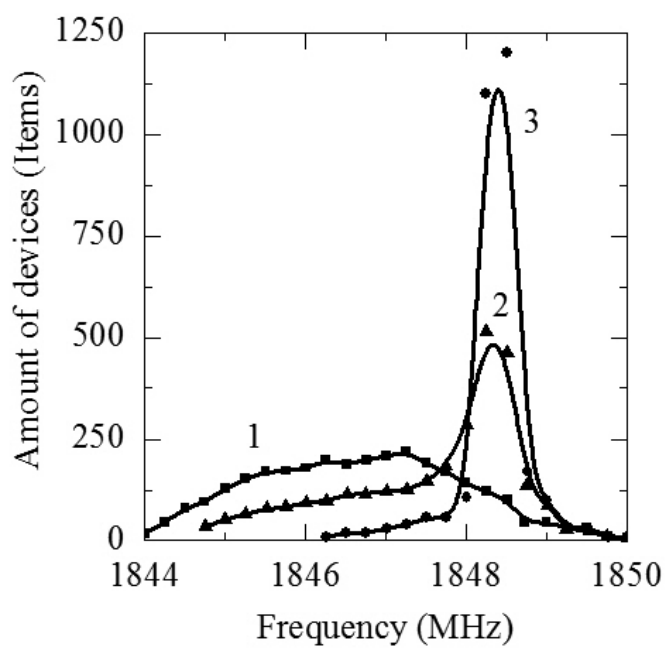

Fig. 4. The effect of the double-step trimming on the production yield of an AlN capped temperature compensated SAW device with the required frequency range: $1-$ the initially deposited layer before the trimming process; 2 after the 1 st trim; 3 - after the 2 nd trim. 
the production yield can be improved by a factor of 20 with double-step trimming.

\section{Summary and conclusions}

AMSystems' three chamber cluster tool with a FALCON ion source is capable of a high-precision and high-speed treatment of large wafers. Stable $\mathrm{SiO}_{2}$ layer can be achieved through high power density UV radiation and annealing in high humidity environment. A uniform capping layer can be achieved by a trimming process involving the FALCON focused ion beam. Ion beam current generated by the FALCON ion source is measured for different pumping rates, providing the possibility for the selection of the ion beam current and the ion energy and prevent overheating of the processed wafer. Experimental measures of the operational frequency of the manufactured SAW devices have shown the efficiency of such a trimming process. As a result of the double-step trimming a total improvement of the production yield of AlN based SAW devices by a factor of 20 was achieved.

\section{References}

1. Mishin, S. (2011). Improving manufacturability of bulk acoustic wave and surface acoustic wave devices. In Joint European Frequency and Time Forum and International Frequency Control Symposium, 9-11 December 2011 (pp. 110-112). EFTF/IFC PP. DOI: 10.1109/SPAWDA.2011.6167203.
2. Willingham, C. B., Parker, T. E., \& Spooner, F. H. (1976). U.S. Patent no. 3,965,444. Washington, D.C.: U.S. Patent and Trademark Office.

3. Jacobs, I. S., \& Bean, C. P. (1963). In G. T. Rado \& H. Suhl (Eds.), Magnetism (Vol. 3, pp. 271-350). New York: Academic Press.

4. Haque, M. S., Naseem, H. A., \& Brown, W. D. (1997). Post-deposition processing of low temperature PECVD silicon dioxide films for enhanced stress stability. Thin Solid Films, 308/309, 68-73. DOI: 10.1016/S0040-6090(97)00542-7.

5. Bizyukov, A. A., Bizyukov, I. A., Girka, O. I., Sereda, K. N., Sleptsov, V. V., Gutkin, M., \& Mishin, S. (2011). Ion beam system for nanotrimming of functional microelectronics layers. Problems of Atomic Science and Technology, Seria: Plasma Phys., 1(17), 110-112.

6. Mishin, S., Gutkin, M., Bizyukov, A., \& Sleptsov, V. (2013). Method of controlling coupling coefficient of aluminum scandium nitride deposition in high volume production. In Joint European Frequency and Time Forum and International Frequency Control Symposium (pp. 126-128). EFTF/IFC. DOI: 10.1109/ EFTF-IFC.2013.6702105.

7. Gutkin, M., Bizyukov, A., Sleptsov, V., Bizyukov, I., \& Sereda, K. (2009). U.S. Patent no. 7,622,721 B2. Washington, D.C.: U.S. Patent and Trademark Office.

8. Girka, O., Bizyukov, I., Sereda, K., Bizyukov, A., Gutkin, M., \& Sleptsov, V. (2012). Compact steady-state and high-flux Falcon ion source for tests of plasma-facing materials. Rev. Sci. Instrum., 83(8), 083501. DOI: $10.1063 / 1.4740519$

9. Zhurin, V. V., Kaufman, H. R., \& Robinson, R. S. (1999). Physics of closed drift thrusters. Plasma Sources Sci. Technol., 8(1), R1-R20. DOI: 10.1088/0963-0252/8/1/021. 